\title{
Trends of popularity of cardiac biomarkers: Insights from Google Trends
}

\author{
Giuseppe Lippi, ${ }^{1}$ Camilla Mattiuzzi, ${ }^{2}$ Gianfranco Cervellin ${ }^{3}$ \\ ${ }^{1}$ Section of Clinical Biochemistry, University of Verona; ${ }^{2}$ Service of Clinical Governance, Provincial Agency \\ for Sanitary Services, Trento; ${ }^{3}$ Emergency Department, University Hospital of Parma, Italy
}

\begin{abstract}
This study was aimed at assessing the trend of worldwide popularity, thus likely reflecting usage, of conventional cardiac biomarkers, including cardiac troponins, myoglobin and creatine kinase MB (CK-MB). Google Trends was interrogated using a combination of the three search terms "troponin" AND "myoglobin" AND "CK-MB", with a time limit set between January 1, 2014 (i.e., the oldest searchable year) and present time (i.e., August 13, 2018). The raw data were entered into an Excel worksheet and reported as cumulative Google Trends scores per week for each cardiac biomarker. The popularity score of myoglobin and CK-MB has displayed a significantly decreasing trend since the 2004, whilst that of troponin has exhibited an apparently paradoxical Ushape behavior, with a more pronounced increase during the past 10 years. The correlation between time and cumulative Google searches was significant for all biomarkers, being $r=0.40$ $(\mathrm{P}<0.001)$ for troponin, $\mathrm{r}=-0.45(\mathrm{P}<0.001)$ for myoglobin and $\mathrm{r}=$ $0.79(\mathrm{P}<0.001)$ for $\mathrm{CK}-\mathrm{MB}$. The score of overall Google searches for troponin was approximately 2.5 -fold and 8.5 -fold higher than for myoglobin and CK-MB, respectively. When the analysis was limited to the past ten years, the correlation between time and cumulative Google searches became even stronger for troponin $(\mathrm{r}=$ $0.85 ; \mathrm{P}<0.001)$, remained virtually identical for CK-MB $(r=-0.80$; $\mathrm{P}<0.001)$, whilst it was no longer significant for myoglobin $(\mathrm{r}=$ $0.13 ; \mathrm{P}=0.150$ ). The graphical analysis of Google search frequency also showed that CK-MB appears to be popular in Mexico, Brazil,
\end{abstract}

Correspondence: Giuseppe Lippi, Section of Clinical Biochemistry, University Hospital of Verona, Piazzale L.A. Scuro, 37134 Verona, Italy.

E-mail: giuseppe.lippi@univr.it

Key words: Biomarkers; Troponin; Myoglobin; Creatine kinase MB; Acute myocardial infarction.

Contributions: the authors contributed equally.

Conflict of interest: the authors declare no potential conflict of interest.

Funding: none.

Received for publication: 14 August 2018.

Revision received: 25 September 2018.

Accepted for publication: 3 October 2018.

This work is licensed under a Creative Commons Attribution 4.0 License (by-nc 4.0).

(C) Copyright G. Lippi et al., 2018

Licensee PAGEPress, Italy

Emergency Care Journal 2018; 14:7769

doi:10.4081/ecj.2018.7769
Chile, Japan, Poland and Romania, myoglobin seems popular in Uruguay, Bolivia, Denmark, Kazakhstan and in some African Nations, whilst troponin is mostly predominant in the remaining parts of the world. The results of this study suggest that, despite all available guidelines share the principle that cardiac troponin should be considered the one and only reference biomarker for diagnosing myocardial ischemia, CK-MB and especially myoglobin are still popular worldwide, especially in certain geographic areas.

\section{Introduction}

Although the vast majority of national and international guidelines, ${ }^{1-5}$ along with reiterated scientific claims, ${ }^{6,7}$ strongly discourage the use of biomarkers other than cardiac troponin $\mathrm{I}(\mathrm{cTnI})$ and $\mathrm{T}(\mathrm{cTnT})$ for diagnosing acute myocardial infarction (AMI), evidence persists that myoglobin and creatine kinase isoenzyme $\mathrm{MB}$ (CK-MB) may be still largely available in many clinical laboratories, and would be hence employed by many clinicians for diagnosing AMI around the world. For example, a very recently national survey showed that the use of obsolete cardiac biomarkers, namely myoglobin and CK-MB, is still relatively frequent in Italy, wherein the percentage of facilities still using either of these two biomarkers in combination with cardiac troponins was found be as high as $33 \%$ and $26 \%$, respectively. ${ }^{8}$ Similar data were reported by another recent US-based survey, according to which the combination of cardiac troponins with other biomarkers was as high as $50 \%{ }^{9}$

Google Trends (Google Inc. Mountain View, CA, USA) is a freely searchable Web-based software, which displays the relative frequency over customized time and location of how much a specific search term is interrogated in Google. ${ }^{10}$ The output of the search is a graph, which displays on the $y$ axis the frequency of how one or more terms have been searched in Google, whilst the $x$ axis reports the period of time over which the search has been carried out. The cumulative number of Google searches is then displayed along an arbitrary scale, ranging between 0 and 100. A value of 100 (i.e., the peak) reflects the highest number of Google searches for the term, a value of 50 reflects $50 \%$ the number of Google searches compared to the peak, whilst a value $<1$ mirrors a number of Google searches $<1 \%$ than the peak. The use of Google Trends has now become commonplace in a variety of clinical settings, especially for analyzing epidemiologic trends of diseases and drugs usage, ${ }^{10,11}$ so that is can now be reliably applied also in the field of laboratory medicine, for assessing how much certain biomarkers are searched in Google and, thereby, how much they are popular and used in routine clinical practice.

Therefore, we carried out an original study based on a Google Trends analysis, aimed at assessing the trend of worldwide popularity of conventional cardiac biomarkers including troponin, myoglobin and CK-MB. 


\section{Materials and Methods}

Google Trends was interrogated using a combination of the three search terms "troponin" AND "myoglobin" AND "CK-MB", with a time limit set between January 1, 2014 (i.e., the oldest searchable year) and present time (i.e., August 13, 2018). According to the Google Trends algorithm, search results were rated according to the peak of popularity (i.e., a value of 100) recorded throughout the search period. The raw data were then entered into an Excel worksheet and reported as cumulative Google Trends scores per week. The statistical analysis was based on Spearman's correlation (including 95\% confidence interval; 95\% CI), using Analyse-it (Analyse-it Software Ltd, Leeds, UK). The study was performed in accordance with the Declaration of Helsinki and under the terms of all relevant local legislations.

\section{Results}

The output of the Google Trend search using the keywords "troponin", "myoglobin" and "CK-MB" is shown in Figure 1. The popularity score of myoglobin and CK-MB displayed a clear decreasing trend over time, whilst that of troponin exhibited an apparently paradoxical behavior, resembling a typical U-shape relationship, with a more pronounced increase of Google Trends score during the past 10 years. The Spearman's correlation between time and cumulative Google searches was significant for all biomarkers, being $\mathrm{r}=0.40(95 \% \mathrm{CI}, 0.26$ to $0.51 ; \mathrm{P}<0.001)$ for troponin, $\mathrm{r}=-0.45(95 \% \mathrm{CI},-0.56$ to $-0.33 ; \mathrm{P}<0.001)$ for myoglobin, and $\mathrm{r}=-0.79(95 \% \mathrm{CI},-0.84$ to -0.73 ; $\mathrm{P}<0.001)$ for $\mathrm{CK}-\mathrm{MB}$. Throughout the search period, the score of overall Google searches for troponin (i.e., 11430; $66.9 \%$ of all Google searches) was approximately 2.5 -fold and 8.5 -fold higher than for myoglobin (i.e., 4306; 25.2\% of all Google searches) and CK-MB (i.e., 1355; $7.9 \%$ of all Google searches), respectively. Even more interestingly, when the Google Trends score of each biomarker was reported as percentage of all Google searches for the three biomarkers, a much more consistent trend was observed, with Spearman's correlation between time and Google searches being $\mathrm{r}=0.80(95 \% \mathrm{CI}$, 0.75 to $0.85 ; \mathrm{P}<0.001)$ for troponin, $\mathrm{r}=-0.67(95 \% \mathrm{CI},-0.75$ to $0.58 ; \mathrm{P}<0.001)$ for myoglobin and $\mathrm{r}=-0.73(95 \% \mathrm{CI},-0.79$ to $0.65 ; \mathrm{P}<0.001$ ) for $\mathrm{CK}-\mathrm{MB}$, respectively (Figure 2 ).

When the analysis was limited to the past ten years (i.e., between August 2008 and August 2018), the correlation between time and cumulative Google searches became even stronger for troponin ( $\mathrm{r}=0.85,95 \% \mathrm{CI} 0.80$ to $0.90 ; \mathrm{P}<0.001)$, remained virtually identical for CK-MB ( $\mathrm{r}=-0.80,95 \% \mathrm{CI},-0.86$ to -0.73 ; $\mathrm{P}<0.001)$, whilst it was no longer significant for myoglobin $(\mathrm{r}=$ 0.13 ; $95 \% \mathrm{CI},-0.30$ to $0.05 ; \mathrm{P}=0.150$ ). Over this 10 -year period, the score of Google searches for troponin (i.e., 7953; 69.4\% of all Google searches) further increased to approximately 3.0-fold and 9.7-fold that of myoglobin (i.e., 2689; 23.5\% of all Google searches) and CK-MB (i.e., 816; 7.1\% of all Google searches), respectively.

A graphical analysis of Google search frequency according to worldwide geography is shown in Figure 3. Interestingly, CK-MB appears to be popular in Mexico, in some Latin American countries (e.g., Brazil and Chile), Japan, Poland and Romania, myoglobin seems popular in some other Latin American countries (e.g., Uruguay and Bolivia), Denmark, Kazakhstan and in some African Nations, whilst troponin is mostly predominant in the remaining parts of the world.

\section{Discussion and Conclusions}

Although a large number of AMI guidelines and recommendations have become available at both international and national level, all of which sharing the same straightforward principle that cardiac troponin (either cTnT or cTnI) should be considered the

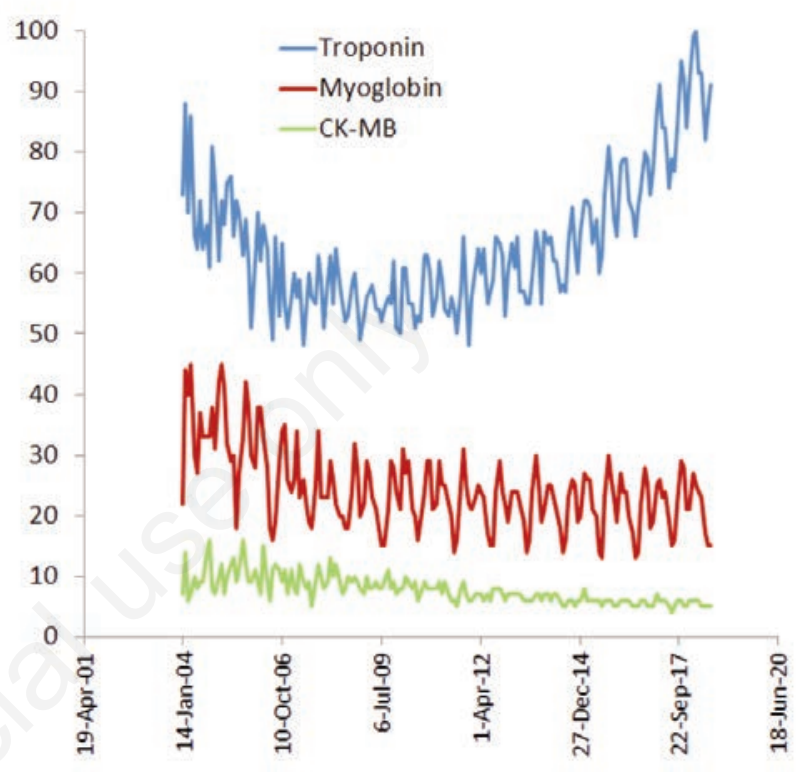

Figure 1. Chronological evolution of cumulative worldwide Google Trends score for the keywords "troponin", "myoglobin" and "CK-MB". CK-MB, creatine kinase isoenzyme MB.

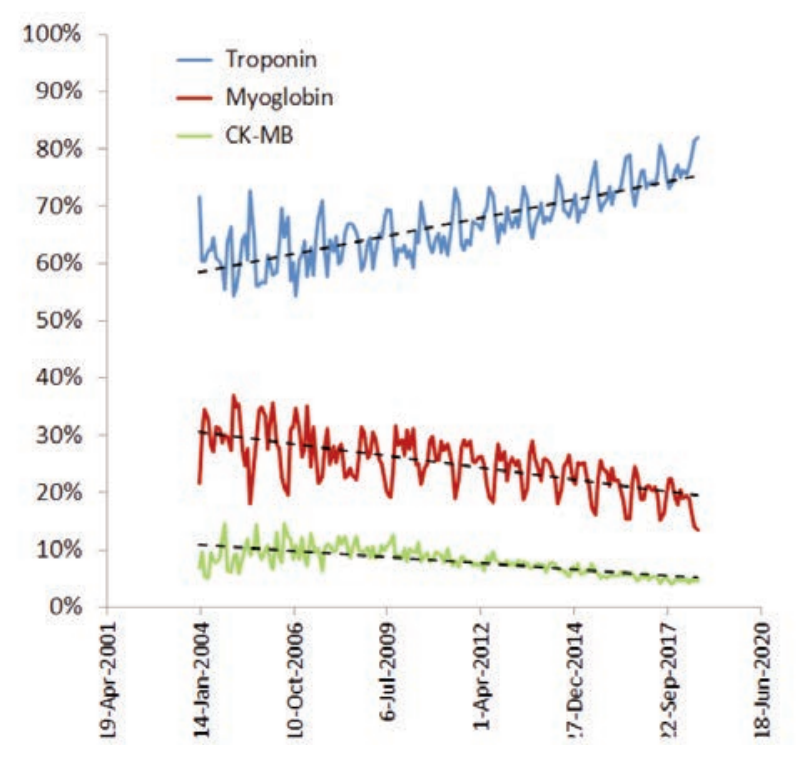

Figure 2. Chronological evolution of percentage worldwide Google Trends score for the keywords "troponin", "myoglobin" and "CK-MB". 
one and only reference biomarker for diagnosing myocardial ischemia, ${ }^{12}$ evidence remains that additional tests, namely myoglobin and CK-MB, may still be used for diagnosing AMI in many healthcare facilities.

The results of our analysis, based on Google Trends, clearly reflects this perception. In agreement with two recent nationwide surveys, ${ }^{8,9}$ we hence showed that despite the popularity of myoglobin and CK-MB has substantially declined over time, nearly onethird of cumulative Google searches still regarded these two biomarkers. Since it has been convincingly demonstrated that Google Trends is a reliable tool for complementing traditional collection and analysis of data used for many healthcare applications, including disease epidemiology, diagnosis and therapy, ${ }^{13-15}$ the findings of our analysis would hence suggest that CK-MB, and especially myoglobin, are still broadly searched (and thereby probably used) biomarkers, especially in certain geographic areas, as those shown in Figure 3. Indeed, the popularity of CK-MB has constantly declined over time, exhibiting a nearly $50 \%$ decline throughout our observational period (i.e., between the years 2004-2018), which was mostly pronounced in the last 10 years. Conversely, the popularity of myoglobin has displayed a consistent decline between 1510 years ago, but has then remained fairly stable during the past 10 years (Figure 1). Notably, the popularity trend of cardiac troponin has exibited a quite paradoxical behavior, reaching its nadir between the years 2004 and 2008, but then constantly growing during the past 10 years (Figure 1). This constantly incremental trend is more clearly perceptible when Google Trends score is reported in percentage of all Google searches (Figure 2) and has virtually coincided with, and then paralleled, the development and commercialization of a new generation of high-sensitive cardiac troponins immunoassays, ${ }^{16,17}$ which have virtually revolutionized the diagnostic approach to patients with suspected AMI, leading to an irreversible obsolescence of whatever else putative cardiac biomarker. These findings are essentially aligned with those presented in other earlier surveys, showing that the so-called obsolete cardiac biomarkers (i.e., myoglobin and CK-MB) are still widely available worldwide, with a frequency of usage that has been reported to be as high as $50 \%{ }^{8,9}$

Some hypotheses can be made for justifying our findings. The first important aspect is perhaps the gap of knowledge on, or adherence to, the most recent AMI guidelines. Several lines of evi-

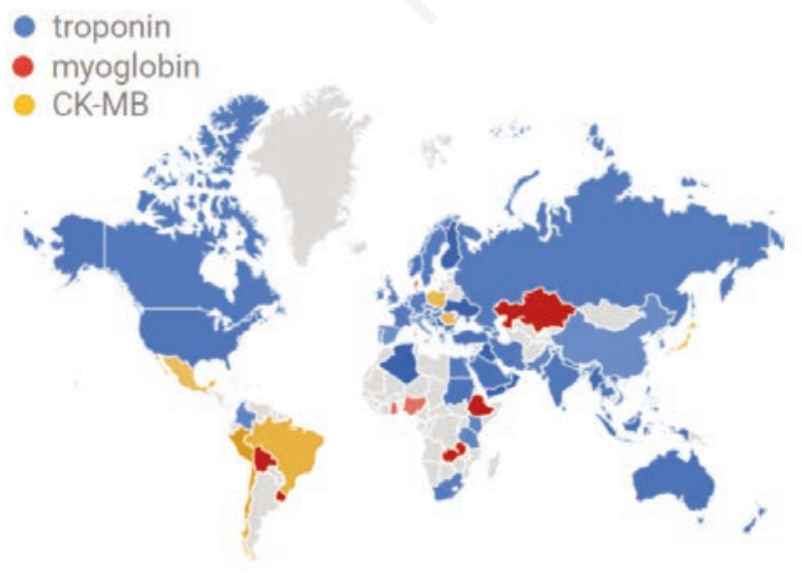

Figure 3. Geographical distribution of the Worldwide Google Trends score for the keywords "troponin", "myoglobin" and "CKMB”. dence now attest that there is a compelling need for both reinforced education of emergency physicians and strengthened adherence to available recommendations in emergency care settings, which would then allow to more efficiently translating skills into clinical practice. ${ }^{18,19}$ The diagnosis of AMI makes no exception to this rule, as recently emphasized by Hachey et al.,${ }^{9}$ who showed that AMI guidelines or recommendations are only followed by less than $40 \%$ of US physicians. This aspect has meaningful clinical implications, as recently highlighted by Shah et al., ${ }^{20}$ who demonstrated that poor adherence to evidence-based management of patients with non-ST elevation myocardial infarction is associated with $10 \%$ higher 3-year mortality after hospital discharge. Notably, laboratory professionals and cardiologists are not exempted from this educational drawback. In particular, Collinson et al. recently showed that an important gap remains between best-practice AMI recommendations and laboratory practice, with many clinical laboratories still providing diagnostic panels including $\mathrm{CK}$ and/or CK-MB and/or myoglobin, ${ }^{21}$ whilst Breuckmann et al. emphasized that noncompliance with AMI guidelines is over $60 \%$ in chest pain units, especially when managing patients with high to intermediate risk. ${ }^{22}$

Defensive medicine is a second important driver of inappropriate requests for obsolete cardiac biomarkers. The use of laboratory resources should always be discretionary, and shall hence be guided by accurate assessment of pre-test probability according to clinical history, signs and symptoms..$^{23}$ However, the real practice is often different from the theory. Many clinicians may be persuaded that the larger is the panel of laboratory tests requested, the less likely is the chance of missing a diagnosis, ${ }^{24,25}$ especially for medical conditions with the highest number of malpractice claims, such as AMI. ${ }^{26}$ This does not hold true, however, as recently emphasized by Alvin et al., ${ }^{7}$ wherein inappropriate or unjustified prescription of obsolete cardiac biomarkers (especially CK-MB) is associated with otherwise preventable healthcare expenditure, no added value for patient care, but also with the risk of generating false positive test results, which might then trigger unwarranted and even invasive diagnostic investigations (e.g., angiography).

Regarding myoglobin, another different explanation may coexist. Unlike CK-MB, which has been almost exclusively used for diagnosing myocardial (prevalently ischemic) injury, myoglobin is currently used for investigating patients with skeletal muscle injuries, especially with rhabdomyolysis. ${ }^{27}$ More specifically, evidence has been brought during the past 10 years that myoglobin may predict the development of acute kidney injury much more efficiently than any other muscular biomarker, including total CK. ${ }^{28}$ Therefore, the Google Trends score recorded for myoglobin may have been artificially inflated by many Google searches focalized on skeletal muscle diagnostics rather than on cardiac testing. This would also explain the lower decrease of Google searches recorded during the last few years, which are those corresponding to the renaissance of this biomarker for diagnosing and monitoring skeletal muscle damage. ${ }^{28}$

We also acknowledge that this study may have some limitations. First, the searches were performed using English terms, and it is hence conceivable that the overall (but not the relative) number of hits may have been biased in some countries where English is not the most spoken language. Notably, we carried out an additional search using either the search terms in English or Italian, but the final trends were almost unvaried (data not shown). Then, the search terms were entered with no reference to the aim of diagnosing myocardial ischemia, since this option is not allowed in Google Trends, and this may have hence contributed to introduce additional variability in our findings. Therefore, the results of our study 
should be regarded as an approximation of the overall popularity of these terms, but do not implicitly reflect the direct usage of these biomarkers.

In conclusion, the results of our study support, and even broaden, previous nationwide information that the worldwide AMI diagnostics is still partially based on obsolete or redundant biomarkers (i.e., myoglobin and CK-MB). Specific educational efforts should hence be planned for promoting the dissemination of currently available AMI guidelines and for preventing unjustified test requests only triggered by defensive medicine.

\section{References}

1. Thygesen K, Alpert JS, Jaffe AS, et al. Third universal definition of myocardial infarction. Circulation 2012;126:2020-35.

2. Casagranda I, Cavazza M, Clerico A, et al. Proposal for the use in emergency departments of cardiac troponins measured with the latest generation methods in patients with suspected acute coronary syndrome without persistent ST-segment elevation. Clin Chem Lab Med 2013;51:1727-37.

3. Roffi M, Patrono C, Collet JP, et al. 2015 ESC Guidelines for the management of acute coronary syndromes in patients presenting without persistent ST-segment elevation: Task Force for the Management of Acute Coronary Syndromes in Patients Presenting without Persistent ST-Segment Elevation of the European Society of Cardiology (ESC). Eur Heart J 2016;37:267-315.

4. Mueller C, Giannitsis E, Möckel M, et al. Rapid rule out of acute myocardial infarction: novel biomarker-based strategies. Eur Heart J Acute Cardiovasc Care 2017;6:218-22.

5. Lippi G, Panteghini M, Bernardini S, et al. Laboratory testing in the emergency department: an Italian Society of Clinical Biochemistry and Clinical Molecular Biology (SIBioC) and Academy of Emergency Medicine and Care (AcEMC) consensus report. Clin Chem Lab Med 2017 Mar 31. doi: 10.1515/cclm-2017-0077. [Epub ahead of print].

6. Jaruvongvanich V, Rattanadech W, Damkerngsuntorn W, et al. CK-MB activity, any additional benefit to negative troponin in evaluating patients with suspected acute myocardial infarction in the emergency department. J Med Assoc Thai 2015;98:93541.

7. Alvin MD, Jaffe AS, Ziegelstein RC, Trost JC. Eliminating creatine kinase-myocardial band testing in suspected acute coronary syndrome: a value-based quality improvement. JAMA Intern Med 2017;177:1508-12.

8. Galli GA, Malloggi L, Moretti M, et al. The IV survey of GdS MM SIPMeL: state of the art of "necrosis" cardiac markers in Italy. Riv Ital Med Lab 2018;14:87-96.

9. Hachey BJ, Kontos MC, Newby LK, et al. Trends in use of biomarker protocols for the evaluation of possible myocardial infarction. J Am Heart Assoc 2017;6:e005852.

10. Cervellin G, Comelli I, Lippi G. Is Google Trends a reliable tool for digital epidemiology? Insights from different clinical settings. J Epidemiol Glob Health 2017;7:185-9.

11. Lippi G, Mattiuzzi C, Cervellin G, Favaloro EJ. Direct oral anticoagulants: analysis of worldwide use and popularity using
Google Trends. Ann Transl Med 2017;5:322.

12. Cervellin G, Mattiuzzi C, Bovo C, Lippi G. Diagnostic algorithms for acute coronary syndrome-is one better than another? Ann Transl Med 2016;4:193.

13. Nuti SV, Wayda B, Ranasinghe I, et al. The use of google trends in health care research: a systematic review. PLoS One 2014;9:e109583.

14. Schootman M, Toor A, Cavazos-Rehg P, et al. The utility of Google Trends data to examine interest in cancer screening. BMJ Open 2015;5:e06678.

15. Tkachenko N, Chotvijit S, Gupta N, et al. Google Trends can improve surveillance of Type 2 diabetes. Sci Rep 2017;7:4993.

16. Galli C, Lippi G. High-sensitivity cardiac troponin testing in routine practice: economic and organizational advantages. Ann Transl Med 2016;4:257.

17. Lippi G, Cervellin G, Sanchis-Gomar F. Critical appraisal to using relative or absolute cardiac troponins change for diagnosing acute myocardial infarction. J Lab Precis Med 2018;3:43.

18. Ebben RH, Vloet LC, Verhofstad MH, et al. Adherence to guidelines and protocols in the prehospital and emergency care setting: a systematic review. Scand J Trauma Resusc Emerg Med 2013;21:9.

19. Venkatesh AK, Savage D, Sandefur B, et al. Systematic review of emergency medicine clinical practice guidelines: Implications for research and policy. PLoS One 2017;12:e0178456.

20. Shah BR, O'Brien EC, Roe MT, et al. The association of inhospital guideline adherence and longitudinal postdischarge mortality in older patients with non-ST-segment elevation myocardial infarction. Am Heart J 2015;170:273-80.

21. Collinson P, Hammerer-Lercher A, Suvisaari J, et al. How well do laboratories adhere to recommended clinical guidelines for the management of myocardial infarction: The CARdiac MArker Guidelines Uptake in Europe study (CARMAGUE). Clin Chem 2016;62:1264-71.

22. Breuckmann F, Hochadel M, Darius H, et al. Guideline-adherence and perspectives in the acute management of unstable angina - Initial results from the German chest pain unit registry. J Cardiol 2015;66:108-13.

23. Lippi G, Cervellin G, Plebani M. The ten commandments of laboratory testing for emergency physicians. Clin Chem Lab Med 2014;52:183-7.

24. Lippi G, Mattiuzzi C. The biomarker paradigm: between diagnostic efficiency and clinical efficacy. Pol Arch Med Wewn 2015; 125:282-8.

25. Lippi G, Mattiuzzi C, Cervellin G. Biomarker validation in the emergency department. General criteria and clinical implications. Emerg Care J 2014;10:1860.

26. Bianchin C, Prevaldi C, Corradin M, Saia M. Medical malpractice claim risk in emergency departments. Emerg Care J 2018; $14: 24-7$ s.

27. Cervellin G, Comelli I, Benatti M, et al. Non-traumatic rhabdomyolysis: Background, laboratory features, and acute clinical management. Clin Biochem 2017;50:656-62.

28. Lippi G, Plebani M. Serum myoglobin immunoassays: obsolete or still clinically useful? Clin Chem Lab Med 2016;54: 1541-3. 